\title{
A NOTE ON THE PARALLELIZABILITY OF REAL STIEFEL MANIFOLDS
}

\section{DAVID HANDEL ${ }^{1}$}

1. Introduction. Sutherland has proved in [4], among other things, that the Stiefel manifolds $V_{n, q}$ of orthonormal $q$-frames in $R^{n}$ are parallelizable for $q \geqq 2$. The proof there consists of showing that the $V_{n, q}$ are stably parallelizable, and then invoking some results of Adams and Kervaire, [1], [2], and [3], which show that under certain circumstances stably parallelizable implies parallelizable. The purpose of this note is to give a more elementary proof of the parallelizability of the $V_{n, q}$ for $q>2$.

2. Preliminaries and statement of the theorem. Let $S^{n-1}$ denote the unit sphere in $R^{n}$. $V_{n, q}$ can then be regarded as the subspace of $S^{n-1} \times \cdots \times S^{n-1}, q$ times, consisting of all $\left(x_{1}, \cdots, x_{q}\right)$ in $S^{n-1}$ $\times \cdots \times S^{n-1}$ with $x_{i} \perp x_{j}$ for $i \neq j$. As such, $V_{n, q}$ is a differentiable submanifold of $S^{n-1} \times \cdots \times S^{n-1}$.

Theorem. $V_{n, q}$ has a trivial normal bundle in $S^{n-1} \times \cdots \times S^{n-1}$. If $q>2$, then $V_{n, q}$ is parallelizable.

For any bundle $\xi$, write $\xi_{x}$ for the fibre over a point $x$ in the base space. Write $\bar{\tau}$ for the tangent bundle of $S^{n-1} \times \cdots \times S^{n-1}, \tau$ for the tangent bundle of $V_{n, q}$ and $\nu$ for the normal bundle of $V_{n, q}$ in $S^{n-1}$ $\times \cdots \times S^{n-1}$. Then $\bar{\tau} \mid V_{n, q}=\tau \oplus \nu$, where $\oplus$ denotes Whitney sum.

If $x=\left(x_{1}, \cdots, x_{q}\right) \in S^{n-1} \times \cdots \times S^{n-1}, \bar{\tau}_{x}$ consists of all $\left(x ; u_{1}, \cdots, u_{q}\right)$ where $u_{i} \in R^{n}, u_{i} \perp x_{i}, 1 \leqq i \leqq q$. The inner product of $\left(x ; u_{1}, \cdots, u_{q}\right)$ and $\left(x ; u_{1}^{\prime}, \cdots, u_{q}^{\prime}\right)$ in $\bar{\tau}_{x}$ is $\sum_{i} u_{i} \cdot u_{i}^{\prime}$. For $x \in V_{n, q}, \tau_{x}$ is the orthogonal complement of $\nu_{x}$ in $\left(\bar{\tau} \mid V_{n, q}\right)_{x}$.

3. The normal bundle $\nu$. If $x \in V_{n, q}$, then $\left(x ; u_{1}, \cdots, u_{q}\right)$ $\in\left(\bar{\tau} \mid V_{n, q}\right)_{x}$ is normal to $V_{n, q}$ if and only if

$$
\lim _{x^{\prime} \rightarrow x ; x^{\prime} \in V_{n, q}} \frac{\left(x-x^{\prime}\right) \cdot\left(u_{1}, \cdots, u_{q}\right)}{\left\|x-x^{\prime}\right\|}=0 .
$$

For $1 \leqq r<s \leqq q$, let $\lambda_{r, 8}$ denote the line subbundle of $\bar{\tau} \mid V_{n, q}$ whose fibre $\left(\lambda_{r, s}\right)_{x}$ is spanned by

Received by the editors September 6, 1964.

1 The author held a National Science Foundation Summer Fellowship for Graduate Teaching Assistants during the preparation of this note. 


$$
\underset{r \text { th }}{\left(x ; 0, \cdots, 0, x_{s}, 0, \cdots, 0, x_{r}, 0, \cdots, 0\right) .}
$$

$\lambda_{r, 8}$ is a trivial bundle, and for $(r, s) \neq\left(r^{\prime}, s^{\prime}\right),\left(\lambda_{r, 8}\right)_{x}$ is orthogonal to $\left(\lambda_{r^{\prime}, s^{\prime}}\right)_{x}$ for all $x \in V_{n, q}$. Thus, $\oplus_{1 \leqq r<s \leqq q} \lambda_{r, 8}$ is a trivial $\frac{1}{2} q(q-1)$ plane subbundle of $\bar{\tau} \mid V_{n, q}$.

If $x=\left(x_{1}, \cdots, x_{q}\right)$ and $x^{\prime}=\left(x_{1}^{\prime}, \cdots, x_{q}^{\prime}\right)$ are in $V_{n, q}$, then using the fact that $x_{i} \cdot x_{j}=x_{i}^{\prime} \cdot x_{j}^{\prime}=\delta_{i j}$, we have

$$
\begin{aligned}
& \left|\frac{\left(x-x^{\prime}\right) \cdot\left(0, \cdots, 0, x_{s}, 0, \cdots, 0, x_{r}, 0, \cdots, 0\right) \mid}{\left\|x-x^{\prime}\right\|}\right| \\
& =\frac{\left|\left(x_{r}-x_{r}^{\prime}\right) \cdot x_{s}+\left(x_{s}-x_{s}^{\prime}\right) \cdot x_{r}\right|}{\left(\sum_{i}\left\|x_{i}-x_{i}^{\prime}\right\|^{2}\right)^{1 / 2}}=\frac{\left|\left(x_{r}-x_{r}^{\prime}\right) \cdot\left(x_{s}-x_{s}^{\prime}\right)\right|}{\left(\sum_{i}\left\|x_{i}-x_{i}^{\prime}\right\|^{2}\right)^{1 / 2}} \\
& \leqq\left\|x_{r}-x_{r}^{\prime}\right\| \cdot \frac{\left\|x_{s}-x_{s}^{\prime}\right\|}{\left(\sum_{i}\left\|x_{i}-x_{i}^{\prime}\right\|^{2}\right)^{1 / 2}} \leqq\left\|x_{r}-x_{r}^{\prime}\right\| .
\end{aligned}
$$

As $x^{\prime}$ tends to $x, x_{r}^{\prime}$ tends to $x_{r}$, so $\left\|x_{r}-x_{r}^{\prime}\right\|$ tends to 0 . Hence by (1), $\left(\lambda_{r, s}\right)_{x}$ is normal to $V_{n, q}$ at $x$ for all $x \in V_{n, q}, 1 \leqq r<s \leqq q$ and so $\oplus_{1 \leq r<s \leq q} \lambda_{r, 8}$ is a trivial $\frac{1}{2} q(q-1)$-plane subbundle of $\nu$.

But since $V_{n, q}$ is an $S^{n-q}$-bundle over $V_{n, q-1}$, and $V_{n, 1} \cong S^{n-1}$, it follows by induction on $q$ that $V_{n, q}$ is a manifold of dimension $q(n-1)$ $-\frac{1}{2} q(q-1)$. Hence, since $\operatorname{dim} S^{n-1} \times \cdots \times S^{n-1}, q$ times, is $q(n-1)$, it follows that $\nu$ has fibre dimension $\frac{1}{2} q(q-1)$. Hence, $\nu=\oplus_{1_{s}<s \leq q} \lambda_{r, s}$ $\cong \frac{1}{2} q(q-1)$ (we write $k$ for the trivial $k$-plane bundle over $V_{n, q}$ ).

4. The tangent bundle $\tau$. For $1 \leqq i \leqq q$, let $\alpha_{i}$ denote the subbundle of $\bar{\tau} \mid V_{n, q}$ whose fibre $\left(\alpha_{i}\right)_{x}$ consists of all

$$
\begin{gathered}
(x ; 0, \cdots, 0, u, 0, \cdots, 0) \\
i \text { th }
\end{gathered}
$$

where $u \in R^{n}, u \perp x_{k}, 1 \leqq k \leqq q . \alpha_{i}$ is an $(n-q)$-plane bundle. Note that $\left(\alpha_{i}\right)_{x}$ is orthogonal to $\nu_{x}$ in $\left(\bar{\tau} \mid V_{n, q}\right)_{x}, 1 \leqq i \leqq q$.

For $1 \leqq r<s \leqq q$, let $\beta_{r, s}$ denote the line subbundle of $\bar{\tau} \mid V_{n, q}$ whose fibre $\left(\beta_{r, 8}\right)_{x}$ is spanned by

$$
\underset{r \text { th }}{\left(x ; 0, \cdots, 0, x_{s}, 0, \cdots, 0,-x_{r}, 0, \cdots, 0\right) .}
$$

$\beta_{r, 8}$ is a trivial line subbundle of $\bar{\tau} \mid V_{n, q}$. Note that $\left(\beta_{r, s}\right)_{x}$ is orthogonal to $\nu_{x}$, to all $\left(\alpha_{i}\right)_{x}$, and to $\left(\beta_{r^{\prime}, s^{\prime}}\right)_{x}$ for $(r, s) \neq\left(r^{\prime}, s^{\prime}\right)$. Hence $\left(\oplus_{1 \leq i \leq q} \alpha_{i}\right)$ 
$\oplus\left(\oplus_{1 \leq r<s \leq q} \beta_{r, 8}\right)$ is asubbundle of $\tau$ of fibre dimension $q(n-q)$ $+\frac{1}{2} q(q-1)=q(n-1)-\frac{1}{2} q(q-1)=\operatorname{dim} V_{n, q}$. Hence,

$$
\tau=\left(\underset{1 \leqq i \leqq q}{\oplus} \alpha_{i}\right) \oplus\left(\underset{1 \leqq r \leqq s \leqq q}{\oplus} \beta_{r, 8}\right) \cong\left(\underset{i}{\oplus} \alpha_{i}\right) \oplus \frac{1}{2} q(q-1) .
$$

The trivial $n$-plane bundle $V_{n, q} \times R^{n}$ over $V_{n, q}$ splits as the Whitney sum $\alpha \oplus \gamma$ where $\alpha_{x}$ consists of all $(x, u), u \in R^{n}, u \perp x_{k}, 1 \leqq k \leqq q$, and $\gamma_{x}$ consists of all $(x, v), v \in R^{n}, v$ in the span of $x_{1}, \cdots, x_{q} \cdot \gamma$ is a trivial $q$-plane bundle, having the $q$ everywhere linearly-independent cross-sections $s_{i}$ defined by $s_{i}(x)=\left(x, x_{i}\right), 1 \leqq i \leqq q$. Hence, $\alpha \oplus q \simeq n$. Hence, $k \alpha \oplus l$ is trivial if $l \geqq q$, and $k$ is any positive integer.

Each of the $\alpha_{i}$ above is equivalent to $\alpha$. Hence $\tau \cong q \alpha \oplus \frac{1}{2} q(q-1)$. If $q>2, \frac{1}{2} q(q-1) \geqq q$, and so $\tau$ is trivial.

\section{REFERENCES}

1. J. F. Adams, On the non-existence of elements of Hopf invariant one, Ann. of Math. 72 (1960), 20-104.

2. M. A. Kervaire, Courbure intégrale généralisée et homotopie, Math. Ann. 131 (1956), 219-252.

3. - Relative characteristic classes, Amer. J. Math. 79 (1957), 517-558.

4. W. A. Sutherland, $A$ note on the parallelizability of sphere-bundles over spheres, J. London Math. Soc. 39 (1964), 55-62.

University of Chicago 